\title{
Disorders of Lipid Metabolism in Chronic Kidney Disease
}

\author{
Mustafa C. Bulbula Tuncay Dagel ${ }^{\mathrm{b}}$ Baris Afsarc $^{\mathrm{c}}$ Nuray N. Ulusu ${ }^{\mathrm{d}}$ \\ Masanari Kuwabara ${ }^{e}$ Adrian Covic $^{f}$ Mehmet Kanbay ${ }^{b}$ \\ ${ }^{a}$ School of Medicine, Koç University, Istanbul, Turkey; ${ }^{b}$ Department of Medicine, Division of Nephrology, Koc

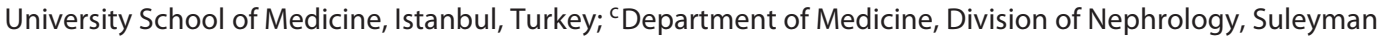 \\ Demirel University School of Medicine, Isparta, Turkey; ${ }^{d}$ Department of Biochemistry, Koc University School \\ of Medicine, Istanbul, Turkey; ${ }^{e}$ Division of Renal Diseases and Hypertension, School of Medicine, University of \\ Colorado Denver, Aurora, CO, USA; ${ }^{f}$ Nephrology Clinic, Dialysis and Renal Transplant Center, "C.I. PARHON" \\ University Hospital, and "Grigore T. Popa" University of Medicine, lasi, Romania
}

\section{Keywords}

Hyperlipidemia • Oxidized low-density lipoprotein · Chronic kidney disease

\begin{abstract}
Cardiovascular disease (CVD) is the leading cause of death in chronic kidney disease (CKD). One of the most important pathophysiological mechanisms for CVD in patients with CKD is the widespread and possibly accelerated formation of atherosclerotic plaques due to hyperlipidemia, uremic toxins, inflammation, oxidative stress, and endothelial dysfunction. Recent studies showed that the level of oxidized lowdensity lipoprotein cholesterol increases, and that highdensity lipoprotein cholesterol dysfunction occurs as kidney function declines and inflammation becomes more prevalent. In this review, we aimed to discuss the effect of kidney dysfunction, oxidative stress, and inflammation on lipid profile.

๑) 2018 S. Karger AG, Basel
\end{abstract}

\section{Introduction}

Cardiovascular disease (CVD) is the leading cause of mortality in Chronic kidney disease (CKD) patients $[1,2]$. Therefore, early determination and management of the risk factors for CVD in CKD patients play an important role to develop more effective screening and treatment strategies to decrease cardiovascular mortality and morbidity in CKD patients. In addition to traditional risk factors for CVD such as older age, male gender, smoking, hypertension, diabetes, and hyperlipidemia, nontraditional risk factors such as anemia, inflammation and oxidative stress, and mineral and bone abnormalities in CKD [3-8] have an undeniable influence on the increased prevalence of CVD observed in CKD. One of the most important pathophysiological mechanisms for CVD in patients with CKD is the widespread and possibly accelerated formation of atherosclerotic plaques due to hyperlipidemia, uremic toxins, inflammation, oxidative stress, and endo-

\section{KARGER}

(C) 2018 S. Karger AG, Basel

E-Mail karger@karger.com

www.karger.com/bpu
Mehmet Kanbay, MD

Koç University School of Medicine

Rumelifeneri Yolu

Sariyer, TR-34450 Istanbul (Turkey)

E-Mail mkanbay@ku.edu.tr, drkanbay@yahoo.com 


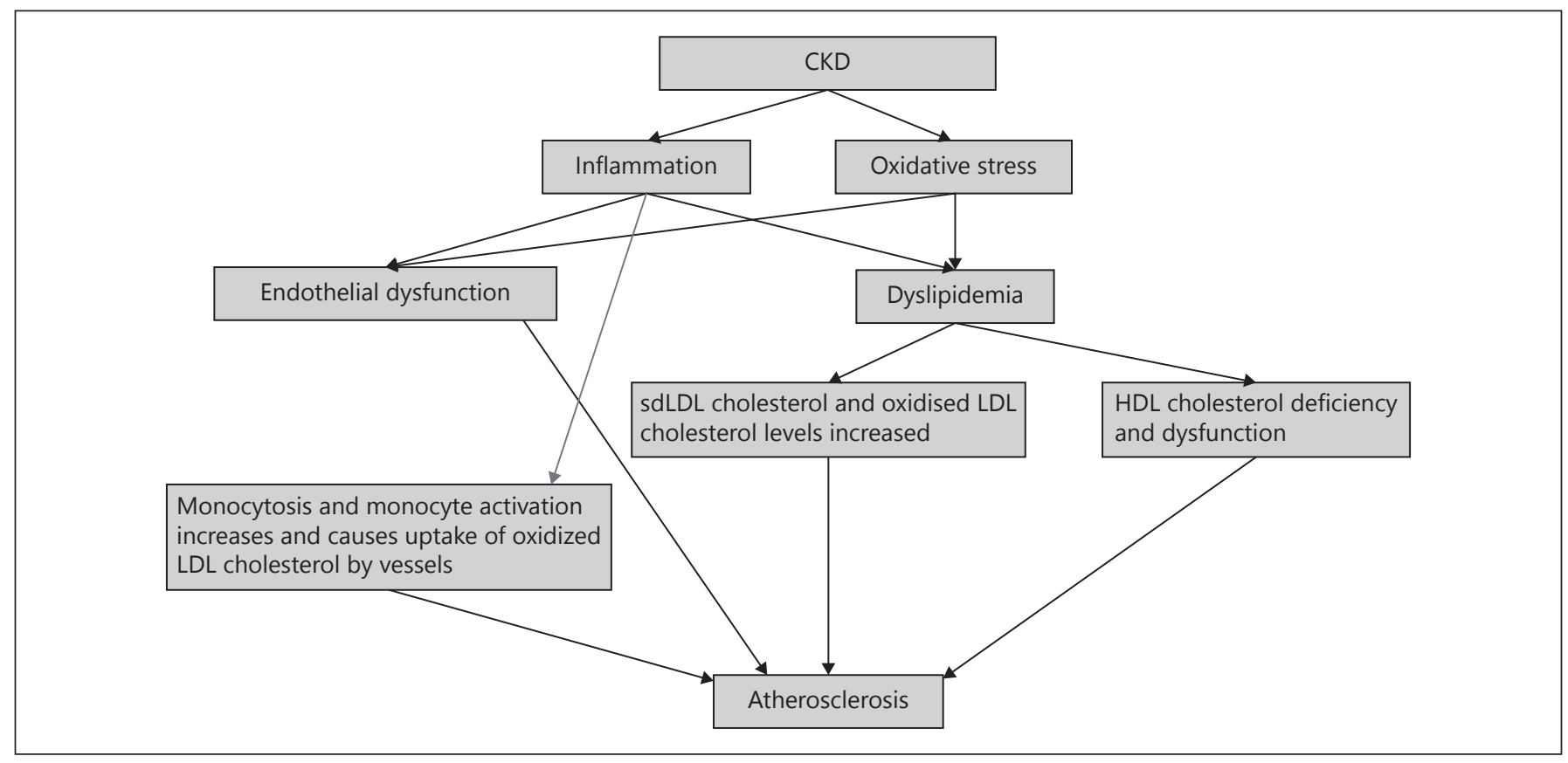

Fig. 1. Development of atherosclerosis in CKD. CKD, chronic kidney disease; LDL, low-density lipoprotein; HDL, high density lipoprotein; sdLDL, small dense LDL.

thelial dysfunction $[9,10]$. Recent studies showed that the level of oxidized low-density lipoprotein (LDL) cholesterol increases and high density lipoprotein (HDL) cholesterol dysfunction occurs as kidney function declines and inflammation becomes more pronounced $[9,11]$ (Fig. 1). In this review, we aimed to discuss the effect of kidney dysfunction, oxidative stress, and inflammation on lipid profile.

\section{An Overview of the Lipoprotein Physiology}

\section{Normal Physiology of Lipoproteins}

Before discussing lipoprotein abnormalities in CKD, a brief explanation of normal lipoprotein physiology is necessary. Lipoproteins are macromolecular particles composed of characteristic lipids and proteins that serve to transport the otherwise insoluble triglycerides and cholesterol molecules. Lipoproteins consist of a coat composed of a monolayer of amphipathic lipids and apolipoproteins (synonym: apoproteins) that surround a core composed of hydrophobic lipids. Since they both have hydrophobic and hydrophilic parts, they can interact with both the aqueous environment of the plasma and the hydrophobic core lipids. Circulating lipoproteins range in size from 5 to $>1,000 \mathrm{~nm}$, and can be separated according to density. HDL are small, containing the least lipid and the most protein, whereas chylomicrons are large and lipid-rich. Lipoproteins are also distinguished by their apolipoprotein contents. Lipoproteins have mainly 2 major functions: One is the delivery of cholesterol and triglyceride molecules from the liver and intestine to muscle and fat tissue via mainly very low-density lipoprotein (VLDL) particles that contain apoB48 and apoB100 or LDL particles. The other function is to assist the transport of excess cholesterol from extrahepatic tissues to the liver for elimination via the bile, which is mediated primarily by HDL particles [12].

\section{Lipoprotein in CKD}

Renal dysfunction is also associated with many perturbations in lipoprotein metabolism leading to dyslipidemia and accumulation of atherogenic particles (Table 1) [11]. CKD is associated with dyslipidemia associating hypertriglyceridemia, elevated LDL cholesterol, an accumulation of apolipoprotein B (Apo B) containing lipoproteins, increased concentrations of lipoprotein(a) particles, and low HDL levels [12]. In CKD, HDL metabolism is impaired and HDL-3 are not maturated into HDL-2 due to a lecithin-cholesterol acyl-transferase (LCAT) de- 
Table 1. Lipid changes in kidney disease

\begin{tabular}{lcc}
\hline Dyslipidemia in CKD & Dyslipidemia in nephrotic syndrome & Lipidomics study-CKD \\
\hline - HDL deficiency and dysfunction & - Increased cholesterol, triglyceride, & - Increased free fatty acid, glycerolipid and \\
- Decreased apoprotein A-1 levels & VLDL, IDL and lipoprotein a levels & glycerophospholipid levels \\
- Increased VLDL, IDL and triglyceride & - Normal HDL, apoprotein A-1 and & - Negative association between eGFR and methyl \\
levels & Apo A-2 levels & hexadecanoic acid and 3-oxooctadecanoic acid \\
- Increased apolipoprotein C-III/C-II & & - Increased palmitic acid and monounsaturated \\
ratio & & level levels and decreased polyunsaturated acid \\
- Normal to increased LDL levels & & \\
- Increased oxidized LDL levels & & \\
\hline
\end{tabular}

ficiency $[13,14]$. In $\mathrm{CKD}$, there is substantial evidence that those oxLDL accumulate, especially in HD patients [15]. All these changes relate to oxidative stress and increased cardiovascular mortality in CKD patients.

\section{Lipoprotein Abnormality in Nephrotic Syndrome}

Dysregulated lipid metabolism leading to dyslipidemia is an often under-recognized, but a nearly universal, complication of persistent nephrotic syndrome [16]. Lipid and lipoprotein metabolism is altered in nephrotic syndrome even without CKD, and the extent of altered lipid metabolism in nephrotic syndrome correlates with the magnitude of proteinuria [17]. The extent of altered lipid metabolism in nephrotic syndrome correlates with the magnitude of proteinuria. In nephrotic syndrome, cholesterol, triglycerides, and Apo B-containing lipoproteins (including VLDL, intermediate-density lipoprotein (IDL) and lipoprotein (a)) are elevated, whereas the concentration of HDL cholesterol and the content of Apo A-I and Apo A-II apolipoproteins are very similar in healthy individuals $[17,18]$. Thus, lipoprotein abnormalities, although are similar with CKD and nephritic syndrome, may also have some differences.

\section{The Significance of HDL Cholesterol Dysfunction and Deficiency in CKD}

Previous studies have affirmed that CKD is an independent risk factor for cardiovascular events, since even slight decreases in the estimated glomerular filtration rate (eGFR) may have deleterious effects on the benefits of HDL cholesterol [19]. HDL cholesterol deficiency and dysfunction in CKD patients play a significant effect on the formation of atherosclerosis through various mechanisms. HDL cholesterol shows anti-oxidant and anti-in- flammatory characteristics, and also hinders the formation of atherosclerosis by decreasing the monocyte infiltration in artery intimal walls [11]. However, HDL cholesterol maturation is impeded due to LCAT deficiency, so that apoprotein (Apo) A-1 level decreases. Furthermore, the release of intracellular cholesterol is limited due to the upregulation of the acyl-CoA cholesterol acyltransferase, and the HDL cholesterol ability to decrease formed oxidized LDL cholesterol levels is restricted due toparaoxonase and glutathione peroxidase deficiency in CKD patients [11].

HDL cholesterol carries the peripheral cholesterol to the liver, which is called reverse cholesterol transport and prevents the cholesterol from being taken up by the macrophages and formation of foamy cells [9]. ApoA-1 deficiency can impair the binding of HDL to ATP binding cassette transporter A-1 and this impaired step causes a dysfunction in free cholesterol efflux from macrophages to HDL cholesterol [9]. The accumulation of free cholesterol in macrophages produces foamy cells in vessels and causes formation of atherosclerotic plaques.

Since atherosclerosis have an inflammatory component, the antioxidant and anti-inflammatory features of HDL cholesterol also contribute to the prevention of atherosclerotic lesions in the vessel wall. HDL cholesterol decreases the production of reactive oxygen species (ROS) and monocyte infiltration by reducing the production of monocyte chemoattractant protein-1 and inhibiting the endothelial adhesion molecule expression [9]. Notably, in CKD patients, HDL cholesterol dysfunction and deficiency impair these anti-oxidant effects and can lead to increased oxidized LDL cholesterol formation [9].

Additionally, the reduction in kidney function causes monocytosis and monocyte activation, which is an additional risk factor for the accelerated atherosclerosis in advanced CKD. Blood monocytes are recruited into the intima and subintimal layers of the vessel wall possibly accounting for the increased uptake of oxidized LDL 
cholesterol accumulation of foam cells [20]. Kanbay et al. [21] investigated the monocyte count/HDL cholesterol ratio alterations in CKD patients. They demonstrated that monocyte/HDL cholesterol ratio increases as the eGFR decreases and monocyte/HDL cholesterol ratio is independent from development of new CVD and mortality in CKD patients.

One of the noteworthy examples of the HDL cholesterol metabolism dysregulation is the one observed in patients with nephrotic syndrome [22]. In patients with nephrotic syndrome, serum HDL cholesterol levels may be normal or below the normal limits, but the ratio of HDL cholesterol to total serum cholesterol is significantly decreased. In nephrotic syndrome, the maturation of cholesterol ester-poor $\mathrm{HDL}_{3}$ to cholesterol ester-rich $\mathrm{HDL}_{2}$ is also defective due to LCAT deficiency as a result of urinary losses. In addition, hypoalbuminemia, which is a characteristic feature of the nephrotic syndrome, contributes to the HDL cholesterol dysfunction, since albumin carries the free cholesterol in the blood to $\mathrm{HDL}_{3}$ in order to decrease the serum cholesterol levels. This also results in an increased level of $\mathrm{HDL}_{3}$ to $\mathrm{HDL}_{2}$ ratio, which can possibly be linked to the formation of the atherosclerotic plaques. Increased cholesterol ester transfer protein levels and reduced hepatic HDL cholesterol docking receptors (SRB1) are additional considerable factors causing HDL cholesterol dysfunction in patients with nephrotic syndrome [22]. As a result, HDL cholesterol deficiency and HDL cholesterol dysfunction may play a crucial role in the development of atherosclerosis. Hence, HDL dysfunction might be an important predictive biomarker for the development and progression of atherosclerosis in $\mathrm{CKD}$. In addition to the benefits of lowering LDL cholesterol levels, improving HDL cholesterol function may also have a positive impact on atherosclerosis progression and risk of a new cardiovascular event, which should therefore be the focus of future studies.

\section{LDL Cholesterol, Intermediate Density Lipoprotein, and Very Low-Density Lipoprotein Cholesterol Dysfunctions in CKD}

In patients with nephrotic syndrome, serum VLDL cholesterol, IDL cholesterol, and triglyceride levels are increased due to impaired urinary clearance, LCAT enzyme, and acquired hepatic LDL receptor dysfunction [23]. Since hepatic lipase has an important function in the removal of the triglyceride content of the IDL cholesterol and conversion of IDL cholesterol to LDL cholesterol, he- patic lipase deficiency in nephrotic syndrome leads to increased serum levels of atherogenic IDL cholesterol and triglyceride enrichment of the LDL cholesterol [17]. Increased apoB-100 levels due to impaired clearance and increased production also result in high LDL cholesterol levels in patients with nephrotic syndrome [17]. Furthermore, Han et al. [23] showed that when compared to the control group, the animals with nephrotic syndrome had significantly increased levels of cholesterol, triglyceride, LDL cholesterol, and free fatty acid synthesized by hepatic tissue.

HDL cholesterol dysfunction with LDL receptor-related protein (LRP) deficiency increases the chylomicron remnant and IDL cholesterol levels and is another factor underlying the formation of small dense LDL (sdLDL) in CKD patients [11]. Although serum LDL cholesterol levels can be normal ranges, sdLDL level - a highly atherogenic subtype of LDL that can be easily oxidized - increases in serum as kidney function worsens [24]. Therefore, both the IDL cholesterol and sdLDL cholesterol can trigger the formation of atherosclerotic plaques even when LDL cholesterol levels are in normal range [24,25]. This also shows the importance of the analysis of detailed lipid profile in patients with CKD.

Serum triglyceride is one of the most valuable lipid types altered in kidney diseases. Hypertriglyceridemia mostly occurs in early stages of CKD [24]. The most dramatic increase in triglyceride levels usually occurs in patients with nephrotic syndrome, but it also increases in other kidney diseases due to both abnormal production and reduced catabolism of triglycerides [24]. Reduced catabolism of triglycerides occurs due to the inactivation of the lipoprotein lipase (LPL). Increased apolipoprotein C-III/C-II ratio precipitates the inactivation of the LPL, since apolipoprotein C-III is an inactivator for LPL, whereas apolipoprotein C-II is an activator for LPL [26]. Chylomicron remnants and IDL cholesterol accumulate because of the decrease in the catabolism of triglycerides [24].

Regarding the analysis of the cholesterol level in CKD patients, it is shown that serum cholesterol level is generally normal or below the normal limit $[24,26]$. However, there is a paradoxical association between serum cholesterol levels and mortality in CKD patients. Low serum cholesterol levels are associated with high mortality in CKD patients due to cardiovascular complications, which can be explained only by increased systemic inflammation and oxidative stress, which may increase oxidized LDL cholesterol level without an increase in LDL cholesterol level in CKD patients $[11,24]$. There- 
fore, lipid profile, especially LDL cholesterol levels with detailed analysis is particularly important to determine the patients' risk for atherosclerosis and other cardiovascular complications due to the capability of the oxidized saddle for accumulation on vessel walls. Cholesterol levels also have an inverse relationship with mortality and needs to be followed up carefully (and triglyceride levels could be used as a biomarker in nephrotic syndrome).

The reverse epidemiology concept is not suggested only for lipids, but this phenomenon has been reported for body weight-for-height measures, systolic and diastolic blood pressures, and serum total cholesterol, LDLcholesterol, homocysteine, creatinine, and parathyroid hormone concentrations [27].

It was shown that low cholesterol along with inflammation predicts morbidity and mortality in hemodialysis patients [28]. However, in non-dialysis CKD patients, recent studies describe a linear relationship between LDLcholesterol and all-cause and cardiovascular mortality $[29,30]$. Thus, reverse epidemiology may be one of the factors explaining the different impact of anti-lipemic therapy in early and late stages of CKD.

\section{The Role of Oxidative Stress and Inflammation in CKD}

One of the important mechanisms considered a risk factor for CVD in patients with CKD is oxidative stress and inflammation. Recent studies indicated that the imbalance between the pro-oxidant and anti-oxidant mechanisms in CKD patients is in favor of increased oxidative stress [31]. Both excessive formation of oxidant compounds and reduced antioxidant enzymes give rise to this condition. Since free radicals have a very short half-life (only seconds), they are difficult to detect in the blood. Therefore, markers of the oxidative stress, such as lipid peroxidation products, protein oxidation, carbohydrate oxidation, and nucleic acid oxidation molecules may be used. Oxidized LDL cholesterol antibodies, $\mathrm{F}_{2}$-isoprostanes, acrolein, and malondialdehyde molecules are the lipid peroxidation products that significantly increase in oxidative stress [31]. In contrast, antioxidant enzymes such as superoxide dismutase, catalase, and glutathione peroxidase levels decrease in CKD patients [32]. Increased oxidant levels in CKD patients are believed to be due to increased uremic toxins [32].

The association between oxidative stress and inflammation is crucially involved in promoting cardiovascular morbidity/mortality in CKD patients [32]. Levels of $\mathrm{F}_{2}$ isoprostanes, which are markers of oxidative stress, are directly correlated with the levels of C-reactive protein, a marker of inflammation [31]. Oxidative stress activates transcription factors that result in the release of proinflammatory cytokines and activation of macrophages. On the other hand, inflammation is also associated with ROS production and induces oxidative stress in tissues [32]. In addition to causing changes in LDL cholesterol function, oxidative stress can also reduce NO activation and synthesis [32] (Fig. 1). Last but not the least, elevated serum ucid acid levels observed in CKD is also associated with lipid disorders [33-37].

\section{Lipidomics in CKD}

In the last decade, many comprehensive research studies have been performed to find new biomarkers to detect early impaired kidney function. Serum creatinine levels are not sensitive and specific to identify early impaired kidney function, since creatinine level is also affected by muscle mass [38]. Lipidomics can be defined as the global study of lipid species and their biological functions within the tissues [38]. In addition to a detailed description of lipoprotein level alterations in patients with CKD, lipidomic analysis may play a critical role in the diagnosis and prognosis of renal diseases as a biomarker and unravel important alterations in the blood levels that may pose a significant risk for the CVD. In a recent study in CKD patients, Chen et al. [39] investigated changes of the lipidomic profile in 180 patients with advanced CKD and 120 age-matched healthy controls and established that free fatty acid, glycerolipid, and glycerophospholipid levels increased in patients with CKD when compared with the control group and also added that triglyceride levels had a positive correlation with free fatty acid, glycerolipid, and glycerophospholipid levels, whereas total cholesterol and eGFR had a negative correlation with these molecular levels. The study also showed that decreased eGFR levels are associated with increased methyl hexadecanoic acid and 3-oxooctadecanoic acid, which are both saturated fatty acids. Since it is thought that increased free fatty acids and saturated fatty acid levels can increase the risk for CVD in CKD patients, these specific fatty acids could be used as a biomarker in CKD patients. In addition to fatty acids, the same study found that reduction in eGFR levels is also linked to an increase in LPC (24:1) and a decrease in PC (20:2/24:1) levels, which are both glyc- 
erophospholipids [39]. Reis et al. [40] stated that phosphadylcholine, plasmenyl ethanolamine, sulfatide, ceramide, and cholesterol sulfate levels can decrease in the LDL cholesterol structure of CKD patients, whereas triacylglyceride and $\mathrm{N}$-acyltaurine levels can increase. Therefore, these changes could cause atherosclerotic plaque formation even though the inflammatory markers and oxidized LDL cholesterol levels are normal.

The study performed in CKD patients undergoing hemodialysis also demonstrated lipid alterations during follow-up. De Gomez et al. [41] found that the palmitic acid and monounsaturated acid levels are increased, whereas polyunsaturated acid levels are reduced during follow-up within 18 months. At the 30th month, the serum levels became more profound. Therefore, recent studies emphasize the importance of lipidomics as a novel biomarker for renal diseases to detect the loss of renal function without any delay. However, further studies with large sample sizes are needed.

\section{Proprotein Convertase Subtilisin/Kexin Type 9 Effect on Lipid Profile}

Proprotein convertase subtilisin/kexin type 9 (PCSK9) is an enzyme also accepted as a new biomarker for lipid metabolism, a novel pharmacological target for hypercholesterolemia, since the inhibition of PCSK9 may be one of the management options to decrease CVD risk $[42,43]$. PCSK9 is primarily synthesized by the liver, intestine, and kidney as a precursor of the $75 \mathrm{kDa}$ protein; it then undergoes autocatalytic cleavage in the endoplasmic reticulum and secreted as a heterodimer protein [43]. PCSK9 causes a reduction in the number of LDL cholesterol receptor by enhancing the degradation of hepatic LDL receptor and this reduces LDL cholesterol uptake leading to a subsequent increase in circulating LDL cholesterol levels $[43,44]$. In addition, recent studies showed that very low-density lipoprotein receptor and apolipoprotein E2 receptor are also partly regulated by PCSK9 [45].

There are contradictory results about the serum PCSK9 level in CKD patients. PCSK9 levels were found to be almost 2 times higher in CKD patients compared with subjects with normal kidney function [46]. In contrast, Rogacev et al. [47] found no correlation between eGFR and PCSK9 levels in CKD patients. Furthermore, PCSK9 concentrations did not predict new CVD events in this study [47]. Lastly, PCSK9 levels are positively correlated with proteinuria in patients with nephrotic syndrome [48].

Lipids in CKD
In both trials, patients with eGFR $<30 \mathrm{~mL} / \mathrm{min} / 1.73 \mathrm{~m}^{2}$ or kidney transplants were excluded. ODYSSEY trials that compared alirocumab with controls (placebo/ezetimibe), mainly as add-on therapy to maximally tolerated statin showed a greater percentage reductions in LDL cholesterol and were associated with a lower incidence of major adverse cardiovascular events [49]. Similarly, in the FOURIER Trial, PCSK9 inhibition with evolocumab significantly reduced LDL cholesterol level and reduced the risk of major adverse limb events in patients with peripheral artery disease. Future studies should be planned to evaluate its effect on outcome in kidney CKD patient [50]. A recent review published by Zeng Lin et al. [51] also examined the 2 novel studies focusing the efficiency of PCSK9 inhibitors.

\section{Treatment Strategies for Dyslipidemia in CKD: How to Improve the Cardiovascular Outcome?}

There are still ongoing studies about the effect of antilipidemic drugs on the progression of CKD and its cardiovascular complications. Although the antilipidemic drugs can normalize lipoprotein levels, the controversial results are found for drug effects on cardiovascular complications. One of the most important studies on this topic, Study of Heart and Renal Protection (SHARP) study, which is a randomized double-blind trial, including 9,270 patients on dialysis showed that simvastatin $20 \mathrm{mg}$ plus ezetimibe $10 \mathrm{mg}$ daily in CKD patients has decreased major atherosclerotic events by $17 \%$ by significantly lowering LDL cholesterol levels during median follow-up of 4.9 years when compared with placebo [52]. Statins are also significantly effective to decrease lipid levels in nephrotic syndrome; however, their long-term effects and their effects on cardiovascular complications should be determined and require further studies [53]. Other than antilipidemic effects, Goicoechea et al. [54] demonstrated the effect of atorvastatin on inflammatory parameters in CKD patients in a 6-month prospective randomized study and they showed that statin therapy can decrease the inflammatory parameters in addition to its anti-lipidemic effects. In addition, Kanbay et al. [55] showed that statins can improve endothelial dysfunction, which occurs due to oxidative stress and significantly decreased systolic and diastolic blood pressure in CKD patients. In contrast, although atorvastatin decreased the serum LDL cholesterol level significantly, Wanner et al. [56] showed that atorvastatin had no statistically significant effect on the cardiovascular death, nonfatal myocardial infarction, and stroke in patients with diabetes receiving hemodialysis [56]. 


\section{Treatment Strategies for Dyslipidemia in CKD: How to Reduce the Rate of Progression in CKD?}

Impaired kidney function can cause dyslipidemia and conversely, it is also demonstrated that altered lipoprotein levels may contribute to the progression of the CKD $[26,55,57]$. Therefore, it is basically thought that abnormal lipoprotein levels should be controlled and treated in CKD patients. Tonelli et al. [58] demonstrated that pravastatin can reduce the rate of renal function loss and slow the decrease in eGFR in CKD patients with proteinuria. Similarly, Bianchi et al. [59] concluded that a combination therapy of atorvastatin with angiotensin converting enzyme inhibitors or angiotensin receptor blockers may be beneficial in slowing down the progression of CKD comorbid with hypercholesterolemia and proteinuria.

Although results are contradictory, the effect of statin therapy on mortality in CKD patients can be related to the stage of CKD. In patients with stages 2-4 CKD, even though statins reduce the LDL cholesterol levels, there is no significant difference in mortality compared to placebo [60]. However, the SHARP trial showed that lipidlowering therapy may improve cardiovascular outcome in CKD patients who are not on dialysis [52]. Seliger et al. [61] showed that statins can decrease the mortality in stage 5 CKD patients, whereas Wanner et al. [56] did not show any survival benefit of statin in stage 5 CKD patients. Other than statins, fibrates are also important components that lead to a decrease in triglyceride levels, especially in nephrotic syndrome and niacin is the most effective drug to increase HDL cholesterol levels. However, their side effects with renal dysfunction could be increased, so further studies about their side effects are needed [60]. As a result, these recent studies are able to create an awareness among people that anti-lipidemics, especially statins, may play a key role to control the progression of the disease, but further research is needed to determine the long-term effects of anti-lipidemics on CKD patients.

Finally, one of the treatment approaches currently in practice is to alleviate oxidative stress and inflammation and thus slowdown the progression of CKD. Antioxidants maybe a significant drug option in CKD patients in the future, but the long-term effects and side effects of the drug are yet to be determined. Recent studies mostly focus on the effects of vitamin E on CKD patients as an antioxidant. Boaz et al. [62] showed that vitamin E can decrease CVDs in hemodialysis patients due to antioxidant effects in randomized, placebo-controlled trials. Al- though these results are promising, the sample size in this study was unfortunately not convincing enough. Islam et al. [63] found that alpha-tocopherol reduces LDL susceptibility to oxidation and has a protective effect against cardiovascular complications in CKD patients on dialysis therapy. It is also demonstrated that vitamin E supplementation can improve the lipid profile in hemodialysis patients [64]. A recent meta-analysis showed that antioxidants may improve early renal damage, but the sample sizes of the studies are small, only a few studies were included for analysis, and the heterogeneity among studies was high [65].

Why statins are not effective in dialysis patients is a subject worth discussing. Although exact causes are not known, various factors may explain these negative results, including advanced stage of disease, low dose of drugs, different pathophysiologic mechanisms of dyslipidemia in CKD patients compared with normal population, predominance of nontraditional cardiovascular risk factors and reverse epidemiology, ignorance of causes of renal failure and the age of the subjects, high dropout rate, and lack of statistical power.

There is still a huge gap in the evidence to support the benefits of antioxidant treatment in delaying the progression of CKD, and further studies have to be performed in order to consider them a legit treatment option. In short, treatment of dyslipidemia and oxidative stress in CKD patients may have a great part in slowing disease progression and reducing cardiovascular complications. Antilipidemic and anti-oxidant drugs could be effectively used after long-term effects are determined.

\section{Conclusion}

In conclusion, cardiovascular complication is a determining factor for morbidity and mortality rate of CKD patients. Chronic kidney dysfunction can cause alterations on lipid profile and these changes mostly form the basis of cardiovascular complications. HDL cholesterol, which is responsible for reverse cholesterol transport, plays an important role to decrease the risk of foamy cells and slows the formation of atherosclerotic plaques in arterial intimal walls. In chronic kidney dysfunction, HDL cholesterol levels decrease and dysfunction occurs due to the loss of molecules for maturation of HDL cholesterol. HDL cholesterol deficiency and dysfunction increase monocyte count that can form foamy cells. HDL cholesterol dysfunction also causes proinflammatory effects and has a role in progression of CKD. Triglyceride levels 
can increase, especially in nephrotic syndrome, due to both increased production and decreased catabolism. The most important alteration on lipid profile may be the changes in composition of LDL cholesterol even LDL cholesterol level could be normal. SdLDL cholesterol or oxidized LDL cholesterol levels increase in CKD due to LRP deficiency, and oxidized LDL cholesterol raises the atherosclerosis risk. However, there is a paradoxical relationship between cholesterol levels and mortality in CKD patients. This shows the predominant effect of oxidative stress and inflammation on mortality in CKD patients. Oxidative stress and chronic inflammation cause endothelial dysfunction, which is a significant risk factor for atherosclerosis. In recent studies, lipidomics has increased the focus on the search for identifying target biomarkers for the early detection of renal dysfunction; however, further research is needed to find more reliable results. Lipidomics could be the new renal biomarker in the near future after more detailed analyses are done. Since dyslipidemia and oxidative stress can also progress renal dysfunction and increase the risk of cardiovascular complications, they can be treated with anti-lipidemics and antioxidant drugs. Statins and vitamin $\mathrm{E}$ are the drug choices that could be effective to both reduce complications and slow the disease progression. Therefore, chronic kidney dysfunction, oxidative stress, and inflammation have a direct relation with dyslipidemia and the formation of atherosclerosis, and markers of those entities can be used for diagnostic and prognostic purposes.

\section{Disclosure Statement}

There are no conflicts of interest for the authors to disclose.

\section{References}

1 Vaziri ND: Dyslipidemia of chronic renal failure: the nature, mechanisms, and potential consequences. Am J Physiol Renal Physiol 2006;290:F262-F272.

2 Zoccali C, Vanholder R, Massy ZA, Ortiz A, Sarafidis P, Dekker FW, et al: The systemic nature of CKD. Nat Rev Nephrol 2017;13: 344-358.

3 Vlagopoulos PT, Sarnak MJ: Traditional and nontraditional cardiovascular risk factors in chronic kidney disease. Med Clin North Am 2005;89:587-611.

4 Kendrick J, Chonchol MB: Nontraditional risk factors for cardiovascular disease in patients with chronic kidney disease. Nat Clin Pract Nephrol 2008;4:672-681.

5 Kanbay M, Vervloet M, Cozzolino M, Siriopol D, Covic A, Goldsmith D, et al: Novel faces of fibroblast growth factor 23 (FGF23): iron deficiency, inflammation, insulin resistance, left ventricular hypertrophy, proteinuria and acute kidney injury. Calcif Tissue Int 2017; 100:217-228.

6 Kanbay M, Solak Y, Siriopol D, Aslan G, Afsar B, Yazici D, et al: Sclerostin, cardiovascular disease and mortality: a systematic review and meta-analysis. Int Urol Nephrol 2016;48: 2029-2042.

7 Yilmaz MI, Siriopol D, Saglam M, Unal HU, Karaman M, Gezer M, et al: Osteoprotegerin in chronic kidney disease: associations with vascular damage and cardiovascular events. Calcif Tissue Int 2016;99:121-130.

-8 Afsar B, Yilmaz MI, Siriopol D, Unal HU, Saglam M, Karaman M, et al: Thyroid function and cardiovascular events in chronic kidney disease patients. J Nephrol 2017;30:235-242.

-9 Moradi H, Vaziri ND, Kashyap ML, Said HM, Kalantar-Zadeh K: Role of HDL dysfunction in end-stage renal disease: a double-edged sword. J Ren Nutr 2013;23:203-206.

-10 Kanbay M, Afsar B, Siriopol D, Unal HU, Karaman M, Saglam M, et al: Endostatin in chronic kidney disease: associations with inflammation, vascular abnormalities, cardiovascular events and survival. Eur J Intern Med 2016;33:81-87.

11 Vaziri ND: Role of dyslipidemia in impairment of energy metabolism, oxidative stress, inflammation and cardiovascular disease in chronic kidney disease. Clin Exp Nephrol 2014;18:265-268.

12 Cohen DE, Fisher EA: Lipoprotein metabolism, dyslipidemia, and nonalcoholic fatty liver disease. Semin Liver Dis 2013;33:380388.

13 Moradi H, Pahl MV, Elahimehr R, Vaziri ND: Impaired antioxidant activity of high-density lipoprotein in chronic kidney disease. Transl Res 2009;153:77-85.

14 Vaziri ND, Liang K, Parks JS: Down-regulation of hepatic lecithin:cholesterol acyltransferase gene expression in chronic renal failure. Kidney Int 2001;59:2192-2196.

15 Ribeiro S, Faria Mdo S, Silva G, Nascimento H, Rocha-Pereira P, Miranda V, et al: Oxidized low-density lipoprotein and lipoprotein(a) levels in chronic kidney disease patients under hemodialysis: influence of adiponectin and of a polymorphism in the apolipoprotein(a) gene. Hemodial Int 2012;16:481-490.

-16 Agrawal S, Zaritsky JJ, Fornoni A, Smoyer WE: Dyslipidaemia in nephrotic syndrome: mechanisms and treatment. Nat Rev Nephrol 2018;14:57-70

17 Vaziri ND: Disorders of lipid metabolism in nephrotic syndrome: mechanisms and consequences. Kidney Int 2016;90:41-52.
8 Joven J, Villabona C, Vilella E, Masana L, Alberti R, Valles M: Abnormalities of lipoprotein metabolism in patients with the nephrotic syndrome. N Engl J Med 1990;323:579-584.

19 Zewinger S, Speer T, Kleber ME, Scharnagl H, Woitas R, Lepper PM, et al: HDL cholesterol is not associated with lower mortality in patients with kidney dysfunction. J Am Soc Nephrol 2014;25:1073-1082.

20 Imhof BA, Aurrand-Lions M: Adhesion mechanisms regulating the migration of monocytes. Nat Rev Immunol 2004;4:432-444.

21 Kanbay M, Solak Y, Unal HU, Kurt YG, Gok $\mathrm{M}$, Cetinkaya $\mathrm{H}$, et al: Monocyte count/HDL cholesterol ratio and cardiovascular events in patients with chronic kidney disease. Int Urol Nephrol 2014;46:1619-1625.

22 Vaziri ND: HDL abnormalities in nephrotic syndrome and chronic kidney disease. Nat Rev Nephrol 2016;12:37-47.

23 Han S, Vaziri ND, Gollapudi P, Kwok V, Moradi $\mathrm{H}$ : Hepatic fatty acid and cholesterol metabolism in nephrotic syndrome. Am J Transl Res 2013;5:246-253.

24 Kwan BC, Kronenberg F, Beddhu S, Cheung AK: Lipoprotein metabolism and lipid management in chronic kidney disease. J Am Soc Nephrol 2007;18:1246-1261.

25 Quaschning T, Krane V, Metzger T, Wanner C: Abnormalities in uremic lipoprotein metabolism and its impact on cardiovascular disease. Am J Kidney Dis 2001;38(4 suppl 1):S14-S19.

26 Nitta K: Clinical assessment and management of dyslipidemia in patients with chronic kidney disease. Clin Exp Nephrol 2012;16:522-529.

27 Kopple JD: The phenomenon of altered risk factor patterns or reverse epidemiology in persons with advanced chronic kidney failure. Am J Clin Nutr 2005;81:1257-1266. 
28 Tsirpanlis G, Boufidou F, Zoga M, Triantafyllis $\mathrm{G}$, Fatourou A, Stamatelou $\mathrm{K}$, et al: Low cholesterol along with inflammation predicts morbidity and mortality in hemodialysis patients. Hemodial Int 2009;13:197-204.

-29 De Nicola L, Provenzano M, Chiodini P, D’Arrigo G, Tripepi G, Del Vecchio L, et al: Prognostic role of LDL cholesterol in non-dialysis chronic kidney disease: multicenter prospective study in Italy. Nutr Metab Cardiovasc Dis 2015;25:756-762.

- 30 Storey BC, Staplin N, Haynes R, Reith C, Emberson J, Herrington WG, et al: Lowering LDL cholesterol reduces cardiovascular risk independently of presence of inflammation. Kidney Int 2017;93:1000-1007.

- 31 Locatelli F, Canaud B, Eckardt KU, Stenvinkel $\mathrm{P}$, Wanner C, Zoccali C: Oxidative stress in end-stage renal disease: an emerging threat to patient outcome. Nephrol Dial Transplant 2003;18:1272-1280.

-32 Vaziri ND: Oxidative stress in uremia: nature, mechanisms, and potential consequences. Semin Nephrol 2004;24:469-473.

- 33 Teng F, Zhu R, Zou C, Xue Y, Yang M, Song $\mathrm{H}$, et al: Interaction between serum uric acid and triglycerides in relation to blood pressure. J Hum Hypertens 2011;25:686-691.

- 34 Peng TC, Wang CC, Kao TW, Chan JY, Yang YH, Chang YW, et al: Relationship between hyperuricemia and lipid profiles in US adults. Biomed Res Int 2015;2015:127596.

35 Berkowitz D: Blood lipid and uric acid interrelationships. JAMA 1964;190:856-858.

36 Kuwabara M, Borghi C, Cicero AF, Hisatome I, Niwa K, Ohno M, Johnson RJ, Lanaspa MA: Elevated serum uric acid increases risks for developing high LDL cholesterol and hypertriglyceridemia: a five-year cohort study in Japan. Int J Cardiol 2018;pii:S0167-5273(17)37927-5.

- 37 Stone NJ, Robinson JG, Lichtenstein AH, Bairey Merz CN, Blum CB, Eckel RH, et al: 2013 ACC/AHA guideline on the treatment of blood cholesterol to reduce atherosclerotic cardiovascular risk in adults: a report of the American College of Cardiology/American Heart Association Task Force on Practice Guidelines. J Am College Cardiol 2014;63(25 Pt B):2889-2934.

38 Zhao YY, Vaziri ND, Lin RC: Lipidomics: new insight into kidney disease. Adv Clin Chem 2015;68:153-175.

39 Chen H, Chen L, Liu D, Chen DQ, Vaziri ND, $\mathrm{Yu} X Y$, et al: Combined clinical phenotype and lipidomic analysis reveals the impact of chronic kidney disease on lipid metabolism. J Proteome Res 2017;16:1566-1578.

-40 Reis A, Rudnitskaya A, Chariyavilaskul P, Dhaun N, Melville V, Goddard J, et al: Topdown lipidomics of low density lipoprotein reveal altered lipid profiles in advanced chronic kidney disease. J Lipid Res 2015;56:413-422.
41 de Gomez Dumm NT, Giammona AM, Touceda LA, Raimondi C: Lipid abnormalities in chronic renal failure patients undergoing hemodialysis. Medicina (B Aires) 2001;61: 142-146.

42 Bergeron N, Phan BA, Ding Y, Fong A, Krauss RM: Proprotein convertase subtilisin/kexin type 9 inhibition: a new therapeutic mechanism for reducing cardiovascular disease risk. Circulation 2015;132:1648-1666.

43 Nozue T: Lipid lowering therapy and circulating PCSK9 concentration. J Atheroscler Thromb 2017;24:895-907.

44 Fisher TS, Lo Surdo P, Pandit S, Mattu M, Santoro JC, Wisniewski D, et al: Effects of $\mathrm{pH}$ and low density lipoprotein (LDL) on PCSK9dependent LDL receptor regulation. J Biol Chem 2007;282:20502-20512.

45 Seidah NG, Awan Z, Chretien M, Mbikay M: PCSK9: a key modulator of cardiovascular health. Circ Res 2014;114:1022-1036.

46 Abujrad H, Mayne J, Ruzicka M, Cousins M, Raymond A, Cheesman J, et al: Chronic kidney disease on hemodialysis is associated with decreased serum PCSK9 levels. Atherosclerosis 2014;233:123-129.

-47 Rogacev KS, Heine GH, Silbernagel G, Kleber ME, Seiler S, Emrich I, et al: PCSK9 plasma concentrations are independent of GFR and do not predict cardiovascular events in patients with decreased GFR. PLoS One 2016; 11:e0146920.

48 Pavlakou P, Liberopoulos E, Dounousi E, Elisaf M: PCSK9 in chronic kidney disease. Int Urol Nephrol 2017;49:1015-1024.

49 Ray KK, Ginsberg HN, Davidson MH, Pordy R, Bessac L, Minini P, et al: Reductions in atherogenic lipids and major cardiovascular events: a pooled analysis of 10 ODYSSEY trials comparing alirocumab with control. Circulation 2016;134:1931-1943.

50 Bonaca MP, Nault P, Giugliano RP, Keech AC, Pineda AL, Kanevsky E, et al: Low-density lipoprotein cholesterol lowering with evolocumab and outcomes in patients with peripheral artery disease: insights from the FOURIER trial (Further Cardiovascular Outcomes Research With PCSK9 Inhibition in Subjects With Elevated Risk). Circulation 2017;137:338-350.

51 Zheng-Lin B, Ortiz A: Lipid management in chronic kidney disease: systematic review of PCSK9 targeting. Drugs 2018;78:215-229.

52 Baigent C, Landray MJ, Reith C, Emberson J, Wheeler DC, Tomson C, et al: The effects of lowering LDL cholesterol with simvastatin plus ezetimibe in patients with chronic kidney disease (Study of Heart and Renal Protection): a randomised placebo-controlled trial. Lancet 2011;377:2181-2192.

53 Kaysen GA: Lipid-lowering therapy in CKD: should we use it and in which patients. Blood Purif 2017;43:196-199.
54 Goicoechea M, de Vinuesa SG, Lahera V, Cachofeiro V, Gomez-Campdera F, Vega A, et al: Effects of atorvastatin on inflammatory and fibrinolytic parameters in patients with chronic kidney disease. J Am Soc Nephrol 2006;17(12 suppl 3):S231-S235.

55 Kanbay M, Yildirir A, Bozbas H, Ulus T, Bilgi M, Muderrisoglu $\mathrm{H}$, et al: Statin therapy helps to control blood pressure levels in hypertensive dyslipidemic patients. Ren Fail 2005;27: 297-303.

56 Wanner C, Krane V, Marz W, Olschewski M, Mann JF, Ruf G, et al: Atorvastatin in patients with type 2 diabetes mellitus undergoing hemodialysis. N Engl J Med 2005;353: 238-248.

57 Kanbay M, Turgut F, Covic A, Goldsmith D: Statin treatment for dyslipidemia in chronic kidney disease and renal transplantation: a review of the evidence. J Nephrol 2009;22:598609.

58 Tonelli M, Moye L, Sacks FM, Cole T, Curhan GC: Effect of pravastatin on loss of renal function in people with moderate chronic renal insufficiency and cardiovascular disease. J Am Soc Nephrol 2003; 14:16051613.

59 Bianchi S, Bigazzi R, Caiazza A, Campese VM: A controlled, prospective study of the effects of atorvastatin on proteinuria and progression of kidney disease. Am J Kidney Dis 2003; 41:565-570.

60 Weiner DE, Sarnak MJ: Managing dyslipidemia in chronic kidney disease. J Gen Intern Med 2004;19:1045-1052.

61 Seliger SL, Weiss NS, Gillen DL, Kestenbaum B, Ball A, Sherrard DJ, et al: HMG-CoA reductase inhibitors are associated with reduced mortality in ESRD patients. Kidney Int 2002; 61:297-304.

62 Boaz M, Smetana S, Weinstein T, Matas Z, Gafter U, Iaina A, et al: Secondary prevention with antioxidants of cardiovascular disease in endstage renal disease (SPACE): randomised placebo-controlled trial. Lancet 2000;356: 1213-1218.

63 Islam KN, O’Byrne D, Devaraj S, Palmer B, Grundy SM, Jialal I: Alpha-tocopherol supplementation decreases the oxidative susceptibility of LDL in renal failure patients on dialysis therapy. Atherosclerosis 2000;150:217224.

64 Daud ZA, Tubie B, Sheyman M, Osia R, Adams J, Tubie S, et al: Vitamin E tocotrienol supplementation improves lipid profiles in chronic hemodialysis patients. Vasc Health Risk Manag 2013;9:747-761.

65 Bolignano D, Cernaro V, Gembillo G Baggetta R, Buemi M, D’Arrigo G: Antioxidant agents for delaying diabetic kidney disease progression: a systematic review and meta-analysis. PLoS One 2017;12:e0178699. 